\title{
Männlichkeit und Verleugnung von Hilfsbedürftigkeit nach berufsbedingten Traumata (Polizei, Feuerwehr, Rettungspersonal)
}

\author{
Masculinity and Avoidance of Help-Seeking after Job-Related Trauma \\ (Police, Firefighters, Rescue Teams)
}

\author{
G. Pieper ${ }^{\mathrm{a}}$ A. Maercker ${ }^{\mathrm{b}}$ \\ ${ }^{a}$ Freie Praxis, Friebertshausen, ${ }^{\text {bKlinische Psychologie und Psychotherapie, TU Dresden }}$
}

\section{Schlüsselwörter \\ Posttraumatische Belastungsstörung · Männlichkeit . \\ Berufsbedingte Traumata - Therapiemotivation}

\section{Zusammenfassung}

Das überwiegend männliche Einsatz- und Rettungspersonal (Polizei, Feuerwehr, Sanitäter, Justizvollzugsbedienstete) zeigt nach berufsbedingten Traumata häufig Schwierigkeiten bei der Akzeptanz von psychotherapeutischen Hilfsangeboten. Im vorliegenden Beitrag wird diese Problematik anhand von Fallbeispielen sowie konzeptuellen Überlegungen des Zusammenhangs zwischen Männlichkeit und Therapiemotivation diskutiert. Klinische Erfahrungen zu männerspezifischen Komplikationen der Posttraumatischen Belastungsstörung (PTBS) und das Modell eines Risikoprofils männlicher Traumaopfer in Einsatzberufen ("Alpha-Männer») werden dargestellt, sowie theoretische Einordnungen in sozialkognitive Theorien der Männlichkeit und Entstehungsmodelle von Traumafolgen vorgenommen. Der Beitrag schließt mit der Skizzierung eines Interventionsvorschlags für berufsbedingte Traumata in den männertypischen Berufsbereichen.

\section{Einleitung}

Viele Jahrzehnte wurde es als selbstverständlich angesehen, daß Polizisten, Feuerwehrleute, Sanitäter, Notärzte und andere Hilfs- und Rettungsdienstmitarbeiter die belastenden Erlebnisse, die sie bei ihrer Tätigkeit erleben, irgendwie alleine verarbeiten. Dabei ging es im Grunde genommen gar nicht um eine «Verarbeitung» des Erlebten, sondern man stellte

\section{Key Words}

Posttraumatic stress disorder - Masculinity .

Profession-related trauma - Treatment motivation

\section{Abstract}

Persons in the predominately male domains of high-risk occupational groups (police, fire department, rescue teams, prison guards) often show difficulties in accepting psychological help after traumatization. The paper presents case reports and conceptual discussion of the relationship between masculinity and treatment motivation. Clinical experiences on male-specific complications of posttraumatic stress disorder (PTSD) and a high risk profile of male work-related trauma victims ('alphaman') are discussed. Theoretical discussion furthermore includes social cognitive theories of masculinity and of development of PTSD. The paper concludes with suggestions for interventions relevant to the outlined problems.

\begin{tabular}{ll}
\hline KARGER & ( ) 1999 S. Karger GmbH, Freiburg \\
Fax +4976145207 14 & Accessible online at: \\
$\begin{array}{l}\text { E-mail Information@Karger.de } \\
\text { www.karger.com }\end{array}$ & www.karger.com/journals/ver
\end{tabular}

sich vor, daß auch die grausamsten Dinge an diesen Männern abprallen würden, daß sie - bildlich ausgedrückt - eine Art Schutzanzug hätten, der sie gegen Traumatisierungen schütze. Erst im Jahre 1994 wurde im DSM IV [American Psychiatric Association, 1994] der Tatsache Rechnung getragen, daß bei einem Teil der Betroffenen die Konfrontation mit einem traumatischen Ereignis, bei dem «intensive Furcht, Hilflosigkeit oder extremer Schreck erlebt wurden», für die Entwicklung 
einer posttraumatischen Belastungsstörung (PTBS) ausreichen.

Seit wenigen Jahren wird in Deutschland von Fachleuten die Notwendigkeit gesehen, Einsatz- und Rettungspersonal nach berufbedingten Traumata therapeutische Hilfen anzubieten [vgl. Bengel, 1997]. Besonders deutlich wurde das nach der ICE-Katastrophe von Eschede im Sommer 1998, als es in der Presse Berichterstattungen gab unter den Überschriften: «Wer hilft den Helfern?», «Hilflose Helfer» oder ähnliches. Dieser Einsicht steht auf der Seite der männlichen Betroffenen mit PTBS oft das Gefühl gegenüber, als «richtiger Mann» keine Angst, keine Hilflosigkeit, keinen Schmerz, keine Trauer, kein Trauma haben zu dürfen, und - wenn es doch so ist daß man sich dieses nicht anmerken lassen dürfe. Das starke, auf selbständiges Handeln ausgerichtete Persönlichkeitsideal, das typischerweise für diese Berufsgruppe prägend ist, kann $\mathrm{zu}$ einer «Verleugnung eigener Hilfsbedürfnisse»[AppelSchumacher, 1997] führen.

Im folgenden sollen einige Fallbeispiele und konzeptuelle Überlegungen zu diesem Thema zur Diskussion gestellt werden. Diese Beispiele entstammen der Arbeit des einen Autors (G.P.) mit Betroffenen der größten Katastrophe im deutschen Braunkohletiefbau, dem Grubenunglück von Borken 1988. Dort wurden bei einer Braunkohleexplosion 51 Bergleute getötet [vgl. Arbeitsgruppe Stolzenbachhilfe, 1992].

Weiterhin wird auf Erfahrungen aus der praktischen Arbeit mit traumatisierten Helfern (Feuerwehrleute, Polizisten) verschiedener schwerer Unglücke und mit Einsatzpersonal bei schwerwiegenden Vorfällen in Justizvollzugsanstalten (Meuterei, Ausbruchsversuche, Geiselnahmen) zurückgegriffen. Es handelt sich um einen klinischen Erfahrungsbericht und nicht um einen wissenschaftlichen Beitrag im Sinne von empirisch überprüften Theorien. Ebenso wird nicht auf die wenigen empirischen geschlechtsvergleichenden Befunde zur PTBS [vgl. Wolfe und Kimerling, 1997] eingegangen, da sich das klinische Anliegen des Beitrags auf Männergruppen bezieht. Der Beitrag berichtet vielmehr von den Erfahrungen, die in langjähriger praktischer Arbeit mit männlichen Helfern bei Unfällen und Katastrophen mit potentiell traumatisierender Wirkung gesammelt wurden und ergänzt diese durch einige theoretische Reflexionen. Die Darstellung verfolgt drei Ziele:

1. Ein größeres Problemverständnis beim einzelnen Notfallhelfer bzw. Therapeuten zu schaffen für die besondere Situation eines traumatisierten Helfers und/oder Helfers mit PTBS, der aufgrund eines rigiden Männerbildes vor einer zusätzlichen Hürde steht, sein Trauma zu verarbeiten. Aufgrund dieser Hürde wird dieser Patient häufig als untherapierbar empfunden («mangelnde Introspektionsfähigkeit», «fehlender Leidensdruck») bzw. er wird als Patient übersehen.

2. Es werden Vorschläge gemacht für ein Rational der Notfallversorgung bzw. ein Therapierational der PTBS, mit denen es leichter möglich wird, einen Zugang zu einem besonders schwierigen und gefährdeten Klientel zu erlangen.
3. Die vorgestellten klinischen Beobachtungen sollen die weitere wissenschaftliche Diskussion anregen, um in diesem Problembereich empirische Forschung zu betreiben (z.B. auf Grundlage der «Emotional Processing Theory» traumatischer Ereignisse von Foa) [vgl. Foa und Kozak, 1998].

\section{Beschreibung einer Problemgruppe mit Fallbeispielen}

Aus der therapeutischen Arbeit mit Katastrophenhelfern des Grubenunglücks von Borken [vgl. Pieper, 1997], der Flugzeugkatastrophe von Ramstein [vgl. Jatzko, 1995] und verschiedenen amerikanischen Untersuchungen [vgl. Ursano, 1994] lassen sich drei Gruppen der in der Mehrzahl männlichen Rettungskräfte beschreiben:

a) Gruppe mit effektivem Bewältigungsverhalten ohne PTBSSymptomatik

Eine Gruppe von Helfern (erfahrungsgemäß die größte), die trotz schwerster Belastungen in einem bestimmten Zeitraum nach dem Trauma keine PTBS-Symptomatik ausgeprägt haben, über adaptive eigene Bewältigungsstrategien verfügen, und gesund bleiben (vgl. SalutogeneseTheorie; z.B. Maercker, 1998).

b) Gruppe mit aktivem Bewältigungsverhalten und PTBS(Teil-)Symptomatik

Eine zweite Gruppe von Helfern, die schwer betroffen und geschockt sind, unter Umständen eine PTBS oder Teilsymptomatik (z.B. Schlafstörungen) ausgebildet haben, die aber handlungsfähig bleiben. Sie suchen Unterstützung entweder im eigenen sozialen Netz oder bei professionellen Notfallhelfern/Traumatherapeuten.

c) Gruppe mit Verleugnung eigener Hilfsbedürfnisse und PTBS-Symptomatik

Eine emotional erschütterte Gruppe von Helfern, die die PTBS-Symptomatik ausgebildet hat. Sie kommen mit den Erlebnissen nicht zurecht, ihre Betroffenheit wird jedoch vor sich und vor anderen geleugnet, da sie nicht mit ihrem Männerbild kompatibel ist.

Im folgenden werden wir die Problemlage der beiden letztgenannten Gruppen näher beleuchten.

\section{Problemlagen}

Einige Aspekte des Zusammenhangs von Traumafolgen und Männlichkeit illustriert das nachfolgende Fallbeispiel eines 32jährigen Mannes (Fall 1):

Herr S. war Mitglied der Grubenwehr und war unmittelbar nach dem Borkener Grubenunglück in die zerstörte und mit giftigen Gasen verseuchte Grube eingefahren. Er mußte etwa 20 der getöteten Kumpel bergen, mit denen er teilweise jahrelang zusammen gearbeitet hatte. Zu den extremsten Streßerfahrungen gehörte das Bergen der Toten, die aussahen, als lebten sie noch, sowie das Bergen und Identifizieren entstellter und zerfetzter Leichen und Leichenteile. 
In den Monaten nach dem Unglück arbeitete er in der Grube weiter, wobei er Aufräumarbeiten erledigte. Nach späteren Aussagen seiner Kumpel erschien er nicht anders als vorher: Er war ein Kollege, der hart anpacken, schwierige und unangenehme Arbeiten erledigen konnte, ohne sich darüber große Gedanken zu machen. Bezüglich des Grubenunglücks tauschte er sich in dieser Zeit mehrfach mit seinen Kameraden aus. Dabei entwickelte sich eine gemeinsame Sprachregelung für die Bewertung der Ereignisse: Man war sich einig, daß das Ereignis schrecklich war, aber man war schließlich ausgebildet für schwierige Einsätze und mußte somit deren Folgen tragen und akzeptieren. Die Kameraden besprachen den technischen Ablauf des Einsatzes, sprachen aber nicht über ihre emotionale Beteiligung und sahen das auch nicht als notwendig an. Typische Gruppensprüche, um die emotionale Beteiligung zu vermeiden waren: «Wenn wir es nicht getan hätten, wer dann?», «Wir sind schließlich dafür ausgebildet...».

Innerlich spürte Herr S. eine immer stärker werdende Unruhe («Ich lief andauernd mit 120\%»). Er litt unter Schlaflosigkeit und Alpträumen und konnte sich nur noch nach Alkoholkonsum einigermaßen entspannen. Bestimmte grauenhafte Szenen seines Einsatzes spielten sich immer wieder vor seinem inneren Auge ab, er empfand es als qualvoll, diese «Filme» nicht stoppen zu können. Gespräche über das Unglück im Familienkreis vermied er so gut es ging. Presse- und Fernsehberichterstattungen über dieses Thema ging er vollkommen aus dem Weg. Er spürte, daß die Erinnerung und Auseinandersetzung damit bei ihm eine Verunsicherung und weitere Unruhe auslöste, bei der er das Gefühl hatte, diese nicht kontrollieren zu können.

Zusätzlich zu den Bildern seiner toten Freunde begann er deren Stimmen zu hören. Er glaubte, sie riefen ihn zu sich. Er fühlte sich schuldig, überlebt zu haben, und er steigerte sich mehr und mehr in den Gedanken hinein, es sei besser, auch tot zu sein. Die Verbindung seines extrem hohen Erregungszustandes, der daraus resultierenden Schlaflosigkeit, den Stimmen und den Alpträumen mit seinem Anspruch «Ich als Kerl und dazu noch als Gruben- und Feuerwehrmann muß so etwas wegstecken!» führte zu einer Spannung, die er nicht mehr bewältigen konnte.

Seine Phantasie, bei seinen toten Freunden sein zu wollen, spitzte sich zu einem konkreten Suizidvorhaben 6 Monate nach dem Grubenunglück zu. Er stand gerade mit dem angeschalteten Fön in der Hand vor der gefüllten Badewanne und wollte einsteigen um sich das Leben zu nehmen, als über seinen Piepser Feuerwehralarm ausgelöst wurde. Er brach sofort sein Vorhaben ab und begab sich - als pflichtbewußter Feuerwehrmann doch noch zum Einsatz. Dies war ein Brand in einem Einfamilienhaus, bei dem er zwei verkohlte Kinderleichen bergen mußte. 3 Stunden nach dem Einsatz brach er zusammen, wurde in eine Klinik eingeliefert und gelangte von dort in die Behandlung innerhalb einer Gruppe der Borkener Katastrophenopfer. In der ersten Zeit der Behandlung ging es fast ausschließlich um die Auseinandersetzung mit seinem Männerbild, die Anerkennung seiner Verletzlichkeit und der nicht zu leugnenden Auswirkungen seines Psychotraumas. Die Phase war mehrfach bedroht von einem Abbruch, da es ihm immens schwer erschien, diese Punkte zu thematisieren.

An diesem Beispielfall werden einige prototypische Problemkonstellationen von Männern in technischen Katastrophenhilfe- und Rettungsberufen deutlich. Der Weg zum Aufsuchen professioneller psychoterapeutischer Hilfe ist dabei meist sehr lang und birgt Gefahren wie die Suizidgefahr in sich. Das Selbstbild des Patienten als «harter Feuerwehrmann» und emotional «unerschütterlicher Retter und Helfer» wird gegen innere Empfindungen und nicht zu leugnende Hinweise seines Körpers (Erregungssteigerung) aufrechtzuerhalten versucht. Der Betroffene kämpft in dieser Phase sozusagen wider besseres Wissen gegen sich selbst, um seinem eigenen Anspruch an Männlichkeit und Zugehörigkeit zur Gruppennorm des harten Mannes zu entsprechen. Die an sich naheliegende Idee, über die belastenden Erfahrungen und inneren Empfindungen mit seiner Frau oder einer Vertrauensperson zu sprechen bzw. sich Hilfe zu suchen, war nicht mit seinem Männer- und Retterideal vereinbar und somit nicht akzeptabel.

Im folgenden werden die häufigsten Problemkonstellationen von traumatisierten Männern in Hilfsberufen, die der oben beschriebenen Gruppe «Verleugnung eigener Hilfsbedürfnisse» zuzuordnen sind, aufgrund klinischer Beobachtungen stichpunktartig dargestellt:

\section{Die PTBS zeigt einen schwierigen Verlauf}

Der schwierigere Verlauf steht damit im Zusammenhang, daß die Betroffenen über längere Zeit die Behandlungsbedürftigkeit ihrer Störung leugnen und sich somit deutlich später in therapeutische Behandlung begeben (siehe Fall oben). Auslöser für den Schritt, doch Fachhilfe in Anspruch zu nehmen, ist häufig eine nicht mehr zu verleugnende Problematik, wie etwa massive Schlafstörungen, sexuelle Funktionsstörungen, unkonzentriertes, unkorrektes Verhalten am Arbeitsplatz oder vollkommene Dekompensation.

\section{Es kommt häufig zur verzögerten Ausbildung eines PTBS- Vollbildes}

Die für verschiedene Traumaarten nachgewiesene verzögerte PTBS-Ausbildung scheint allgemein so abzulaufen, daß nach einem Trauma einzelne PTBS-Symptome bestehen bleiben, die nicht das Vollbild einer PTBS erfüllen. Diese Einzelsymptome bilden sich nicht spontan zurück und in einer neuen, belastenden Lebensphase bzw. -umwelt können dann zusätzliche PTBS-Symptome ausgebildet werden, so daß schließlich ein PTBS-Vollbild entsteht [Maercker, 1998]. Die nach dem Trauma zunächst bestehenden Einzelsymptome können als Hinweisreize aufgefaßt werden, wobei die Betroffenen durch einen günstigen (adaptiven) Bewältigungsstil zur Ausheilung dieser Symptome beitragen können. Bei Männern der beschriebenen Untergruppe von Helfern scheint jedoch die Nichtbeachtung von Hinweisreizen ( = andauernde PTBS-Einzelsymptome) besonders ausgeprägt zu sein. Als Beispiel sei hier der Fall eines 27jährigen Grubenwehrmannes angeführt (Fall 2):

Bei der Borkener Katastrophe war sein Rettungs- bzw. Bergungseinsatz unter Tage für ihn selbst sehr riskant. Zudem war er nach dem Einsatz über Tage mit dem Leid der Angehörigen konfrontiert, was er wegen seiner eigenen Hilflosigkeit äußerst belastend fand. Einige Zeit nach dem Unglück kam er für eineinhalb Jahre zur Bundeswehr. Während dieser Phase hatte er mehrfach Träume vom Katastropheneinsatz bzw. fühlte sich innerlich unruhiger als früher. Nach seiner Bundeswehrzeit wollte er wieder in der Grube arbeiten.

Beim ersten Einfahren in die Grube entwickelte er starke Angstgefühle, hyperventilierte und wehrte sich danach vehement, wieder unter Tage zu arbeiten. Er zeigte das Vollbild einer PTBS mit Hyperarousel, Intrusionen bzw. Flashbacks und Vermeidungsverhalten. 
Er stellte sich bei verschiedenen Ärzten, Fachärzten und einer psychosomatischen Fachklinik vor. Er deutete dabei selbst an, daß seine Probleme etwas mit seinem Einsatz in Borken zu tun haben könnten, bekam jedoch nicht die Diagnose PTBS. Als er 11 Monate später in Kontakt mit dem Behandlungsprogramm für Rettungspersonal kam, stand er unter hoher Tranquilizer-Medikation und hatte das Selbstbild eines gescheiterten Mannes, der sich selbst aufgegeben hat.

Auch bei diesem Grubenwehrmann wirkte die Gruppennorm («Wir stecken so etwas weg») so stark, daß er nicht in der Lage war, seine frühen internen Hinweisreize (Unruhe, weiche Knie, Träume vom Einsatz) ernst zu nehmen und sich nicht traute, anderen mitzuteilen, daß er mit der Verarbeitung der Erlebnisse überfordert war. (Nebenbei gibt der geschilderte Fall einen Hinweis darauf, daß sich auch Ärzte und Psychologen bei Männern aus dem Berufsfeld des Rettungspersonals scheuen können, die Diagnose PTBS zu vergeben.)

\section{Folgen eines Psychotraumas werden durch somatoforme und körperliche Krankheiten manifestiert}

Für traumatisierte Männer in Hilfsberufen stehen die körperlichen Veränderungen im Vordergrund. Das könnte damit zusammenhängen, daß für die Genannten eine typische Schwierigkeit darin liegt, ihre eigene Betroffenheit nicht verbal ausdrücken zu können bzw. eine große Scheu davor zu haben. Die posttraumatische Reaktionstendenz, auf der somatischen Ebene zu reagieren, ist auch in anderen Populationen beschrieben worden, bei denen die Verbalisierung innerer $\mathrm{Zu}$ stände aus verschiedenen Gründen eher unüblich ist (z.B. bosnische Bürgerkriegsflüchtlinge [Butollo, 1997]; Flüchtlinge aus 3.-Welt-Ländern [Kirmayer, 1996]). Bei betroffenen Männern des Grubenunglücks Borken fielen klinisch vor allem auf: Kopf-, Magenschmerzen, allgemeine Müdigkeit und «Zerschlagenheit», häufig «weiche Knie», Herzbeschwerden sowie eine stark verminderte Leistungsfähigkeit.

\section{Allgemein erhöhte Mortalität}

Neben dem unsystematischen Eindruck von Klinikern, daß es insbesondere bei traumatisierten Männern eine raschere Progredienz letaler Krankheiten gibt (z.B. Karzinome, senile Demenzen), liegen erste Hinweise aus Untersuchungen vor, die dies belegen [Baider et al., 1994]. Darüber hinaus weisen epidemiologische Überblicksarbeiten auf eine allgemein erhöhte Mortalität von unbehandelten Traumatisierten hin [vgl. Schnurr, 1996].

Ein Beispiel stammt ebenfalls aus der Borkener Katastrophe, wobei in diesem Fall natürlich keine gesicherte Kausalitätsaussage zu machen ist (Fall 3).

Der 57jährige, in einer Verantwortungsposition stehende Mann mußte nach dem Grubenunglück mitansehen, wie ein getöteter Mitarbeiter nach dem anderen zum Teil erheblich entstellt geborgen wurde. Er mußte sie identifizieren und war durch diese Konfrontation schwer traumatisiert. Er bildete nach der Katastrophe starke Schuldgefühle aus, daß er für Vernachlässigungen der Sicherheitsvorschriften verantwortlich gewesen sei. Die staatsanwaltschaftliche Untersuchung stellte heraus, daß die Kata- strophenursache nicht durch menschliches Versagen verschuldet war. Dennoch litt er weiter unter den starken Schuldgefühlen, daß es in «seiner Ära» zum Tod von 51 Menschen gekommen war.

Einige Monate nach dem Unglück erkrankte er an einem Karzinom und wurde operativ behandelt. Die Angebote zu therapeutischen Gesprächen im Rahmen der Katastrophennachbereitung konnte er nur bedingt annehmen. Er wirkte dabei starr, ließ eine Auseinandersetzung mit seinen Gefühlen nicht zu, sprach aber immer wieder von seiner Schuld. Bald nach der ersten Operation entwickelte sich ein Rezidiv. Bei einem zweiten stationären Aufenthalt erklärte er dem Therapeuten (G.P.), daß er mit den Schuldgefühlen wegen des Unglücks nicht leben könne. Wenige Monate später war er tot.

\section{Anhaltend erhöhte Suizidgefährdung}

Wie das Fallbeispiel 1 illustriert, kommt es bei traumatisierten Männern in erhöhtem Maße zu (para)suizidalen Handlungen. Für diese traumatisierten Männer scheint ein Suizid als Lösungsversuch naheliegender, als eine therapeutische Hilfe in Anspruch zu nehmen. Das Aufsuchen von Hilfe wegen psychischer Probleme bei einem Mitmenschen oder einem Professionellen ist für die Betreffenden gleichbedeutend mit dem Eingeständnis von Schwäche. Die suizidale Handlung läßt sich als extreme Hilflosigkeit und Folge des Fehlens adaptiver Bewältigungsformen verstehen. Traumatisierte Männer in Rettungsdiensten und ähnlichen Berufen scheinen aufgrund einer starren und ausschließlichen Rollenorientierung (siehe unten) besonders prädestiniert dafür zu sein, nach einer Infragestellung ihres Selbstbildes, in ein tiefes «psychisches Loch» zu fallen. Nicht selten entsteht bei den Betroffenen ein Gefühl, haltlos, orientierungslos zu sein und «verrückt» $\mathrm{zu}$ werden. Beispielsweise wird dann direkt geäußert: «Ehe ich ganz verrückt werde, ehe ich in der Klapse lande, bringe ich mich lieber um!»

\section{Verstärkter Alkoholkonsum bis hin zu Alkoholabhängigkeit}

Das Fehlen angemessener Bewältigungsformen erklärt auch die sehr häufig zu beobachtende Tendenz, die durch eine traumatische Erfahrung entstandenen Probleme durch Alkoholkonsum in den Griff zu bekommen. Der unmittelbar erlebte Entspannungseffekt, besonders bezüglich des erhöhten Erregungsniveaus, wirkt dabei positiv verstärkend [vgl. McFarlane, 1998].

Da in den Männergruppen der betreffenden Berufe sowieso häufig nach dem Einsatz - in einer Art ritualisierten Abschaltens - Alkohol getrunken wird («um wieder zur Ruhe zu kommen»), ergibt sich eine besondere Gefährdung zur Abhängigkeitsentwicklung. Dies führt dann auch dazu, daß vor Beginn einer eventuellen Therapie der aktuelle Alkoholabusus erfaßt und dann zunächst eine Entwöhnungskur durchgeführt werden muß.

\section{Höhere Rate von Frühberentungen}

Eine andere Variante der Extremlösungen ist die Möglichkeit der Frühberentungen nach einem traumatischen Erlebnis:

Der in Fall 2 beschriebene Grubenwehrmann mit dem um eineinhalb Jahre verzögerten Beginn einer PTBS war nicht mehr 
in der Lage, unter Tage zu arbeiten, nachdem sich die volle Symptomatik entwickelt hatte. Eine Expositionsbehandlung war wegen seiner mangelnden Motivation damals nicht durchführbar. Er selber erlebte sich als jemand, der überhaupt nicht mehr nach seinen eigenen Wünschen und Ansprüchen funktionierte und hatte sich selbst aufgegeben. Zusätzlich zu seiner PTBS-Symptomatik kamen sexuelle Funktionsstörungen und eine Depression. Im Alter von 29 Jahren sollte er berentet werden.

Neben der positiven Konsequenz des symptomatischen Verhaltens durch eine Berentung, läßt sich der Berentungswunsch auch als «Extremlösung» nach dem Alles-OderNichts-Prinzip erklären, bei dem auch das bisher hochgehaltene Männlichkeitsideal aufgegeben wird.

Als dieser Bergmann sich später doch dazu entschloß, eine Expositionsbehandlung (vor Ort) zu wagen, obwohl er inzwischen durch eine Bergmannsrente finanziell abgesichert war, antwortete er auf die Frage, warum er sich das eigentlich antun wollte: «Weil ich mich wieder als ganzer Mann fühlen möchte! ... Erst wenn ich wieder einfahren kann, fühle ich mich wieder als Mann!»

\section{Theoretische Überlegungen zu den Fallbeispielen}

\section{Modell eines Risikoprofils für posttraumatische Komplikationen}

In diesem Abschnitt soll diskutiert werden, ob die genannten klinischen Erfahrungen bei Männern in Rettungsdiensten und ähnlichen Berufen im Modell des Risikoprofils «AlphaMann» zusammengefaßt werden können. Die Bezeichnung Alpha-Mann (A-M) folgt dabei dem Begriff der Alpha-Position in der Verhaltensforschung, durch den Führungsposition in Rangordnungen bezeichnet wird. Dieser Begriff wurde hauptsächlich deshalb gewählt, weil er sich in ein therapeutisches Rational einbauen läßt, das den Betroffenen gut vermittelbar ist (möglicher «Wiedererkennungseffekt» bei den Betroffenen) und sie dadurch für die Zusammenarbeit mit dem Therapeuten motiviert. (Der Begriff erkennt implizit die Führungspositionen des einzelnen Betroffenen im Rettungsgeschehen während Katastrophen an.)

Das Risikoprofil von «Alpha-Männern» sieht folgendermaßen aus:

1. Sie gehören einer Berufs- oder Freiwilligen-Gruppe an, wie z.B. Polizei, Feuerwehr, Grubenwehr, Personenschützer, Bundeswehr, Rettungssanitäter;

2. sie leben mit dem Selbstbild bzw. Selbstideal eines harten Mannes, den nichts erschüttern kann;

3. sie lehnen es als unmännlich ab, über Gefühle zu reden, bzw. leugnen im allgemeinen, Gefühle wie Angst, Betroffenheit usw. überhaupt zu verspüren;

4. sie sehen sich mit ihrer Gruppe als eine Art Elite mit sehr hohen Gruppenidealen und identifizieren sich mit diesen.

\section{Theoretischer Erklärungsversuch}

Welche Erklärungen lassen sich für dieses Profil, das in Folge eines Traumas ein Risiko für die PTBS-Entwicklung darstellen könnte, heranziehen? Die nachfolgenden Punkte erheben keinen Anspruch auf Vollständigkeit. Ihre jeweilige Wertigkeit für die Erklärung des Risikoprofils ist bisher nur spekulativ, weil empirische Untersuchungen zu diesem Themenkomplex noch ausstehen.

\section{Maskulines Rollenverständnis}

Sozialpsychologische Studien belegen, daß es ein klassisches männliches Rollenstereotyp in westlichen Gesellschaften gibt: Danach werden Unabhängigkeit, Macht, Stärke, Unerschütterlichkeit und Leistung als maskuline Qualitäten definiert [Alfermann, 1996; Sieverding, 1997]. Dieses Rollenstereotyp ist eine normative gesellschaftliche Erwartung, die sich auf wünschenswert angesehene Eigenschaften, Fähigkeiten und Verhaltensweisen bezieht. Die Geschlechtsrollenstereotype schlagen sich nicht direkt im Verhalten einer Person nieder; vielmehr ist das Selbstkonzept als vermittelnde Variable zu berücksichtigen. D.h., Männer unterscheiden sich nach dem Grad der Ähnlichkeit ihres Selbstkonzeptes mit dem typischen maskulinen Rollenstereotyp. In der sozialpsychologischen Literatur wurde vorgeschlagen, die Männer des extrem maskulinen Typs als «Malboro-Mann-Prototyp» zu bezeichnen [Sieverding, 1997]. Dieser Marlboro-Mann verkörpert Eigenschaften wie Unabhängigkeit, sicheres Auftreten, Angstlosigkeit, Stärke, Coolness und Rauhheit. Bei A-M scheint das Selbstkonzept ebenfalls diesem extrem maskulinen Pol zu entsprechen. Damit ist allerdings noch nicht gesagt, ob im individuellen Fall das Wunsch-Selbstkonzept («Marlboro-Prototyp») und das reale Selbstkonzept (bzw. «Selbst») des Betreffenden identisch sind. Nach sozialkognitiven Theorien wird eine Diskrepanz zwischen Prototyp und Selbst angenommen [Cantor et al., 1982]. Wenn diese Diskrepanz eher groß ist, d.h. der Betreffende ein Marlboro- bzw. Alpha-Mann sein möchte, diesem in seinem Erleben und Verhalten allerdings nicht entspricht, stellt dies eine psychosoziale Vulnerabilitätskonstellation dar [vgl. Sieverding, 1997].

\section{Soziales Lernen}

Die Ausgestaltung des männlichen bzw. des extrem männlichen (A-M) Rollenverhältnisses findet in der lebenslangen Sozialisation statt. Diese beginnt in der Kindheit, wenn Jungen und Mädchen in der Regel ein Modellernen bei ihren jeweiligen Geschlechtsvertretern präferieren. Zusätzlich kommt es zu differentiellen Bekräftigungen, bei denen beispielsweise Jungen für geschlechtstypisches Verhalten belohnt und für geschlechtsuntypisches Verhalten bestraft oder ignoriert werden [Alfermann, 1996; Bussey und Bandura, 1984]. Ein aktuelles Beispiel von der ICE-Katastrophe von Eschede kann diesen Mechanismus der differentiellen Bekräftigung illustrieren.

Ein 13jähriger Junge, der im Zug gewesen war, wurde bei der Bahnkatastrophe mittelschwer verletzt. Ein fachkundiger Helfer, der ihn an der 
Unfallstelle betreute und ihn zu einem Unfallarzt brachte, riet ihm, später psychotherapeutische Hilfe in Anspruch zu nehmen. Der Unfallarzt hatte das mitbekommen und sagte zu dem Jungen: «Mach das niemals! Du wirst Dein Leben lang gestempelt sein und außerdem bist Du doch schon ein Mann, das schaffst Du auch so!»

Theorie der erschütterten Grundannahmen durch ein Trauma Die Theorie der erschütterten Grundannahmen (shattered assumption) [Janoff-Bulman, 1985] wurde bisher nicht geschlechtsdifferentiell formuliert. Da sie allerdings ebenfalls eine sozialkognitive Theorie ist, läßt sie sich gut auf die bisher skizzierten Prozesse anwenden. Nach Janoff-Bulman [1985] kann ein Trauma die lebenstragenden Vorannahmen und Erwartungen einer Person über die Welt erschüttern oder zerstören. Typische Vorannahmen sind die eigene Unverwundbarkeit, die Auffassung, daß die Welt verstehbar ist, die Überzeugung, das Selbst ist wertvoll sowie die Annahme, daß man anderen Menschen trauen kann.

Diese lebenstragenden Vorannahmen werden durch eine traumatische Erfahrung schwer erschüttert. Selbstzweifel, Gefühle der Unsicherheit, der permanenten Bedrohung, der Wertlosigkeit und der Schwäche können auftauchen. Die Welt wird als bedrohlich wahrgenommen, die Wahrscheinlichkeit, daß noch einmal ein dramatisches Ereignis eintritt, wird als hoch eingeschätzt.

Eine geschlechtsdifferentielle Ausgestaltung dieser Theorie kann zunächst an den geschlechtsrollentypischen Welt- und Selbstbildern ansetzen (s. oben). Demnach ist das maskuline Rollenstereotyp durch Unabhängigkeit, Macht, Stärke, Unerschütterlichkeit und Leistung gekennzeichnet. Für die AlphaMänner in den Rettungs- und Hilfsberufen gehört zum Weltbzw. Selbstbild, daß ein Polizist/Feuerwehrmann/Retter/Helfer in der Lage ist, immer handlungsfähig zu sein und die Kontrolle zu behalten und daß er jede Aufgabe durch entsprechende Übung und optimale technische Ausstattung bewältigen kann. Daraus können sich Erwartungskonsequenzen ergeben, unter anderem daß man aufgrund seiner Männlichkeit in der Lage ist, anderen in Katastrophenfällen zu helfen, ohne dabei selbst seelisch verwundet oder verletzt zu werden. Das Erleben eines Traumas führt zu einer akuten Diskrepanz zwischen Männlichkeitsprototyp und einem erschütterten Selbst- und Weltbild. Die Erschütterung der Basisannahmen über die Männlichkeit («Ich bin immer handlungsfähig und habe alles unter Kontrolle») wird gleichgesetzt mit dem Verlust von Männlichkeit. Da für viele A-M kein anderes positiv bewertetes Bild von Mann-Sein vorhanden und abrufbar ist, kann dies zu Zuständen der Orientierungslosigkeit und Hilflosigkeit führen, die für viele A-M nicht zu verkraften sind. Wenn alles das, was sie positiv abgehoben hat von Männern, «die keine richtigen Männer sind», nicht mehr stimmt, kann es zu den oben geschilderten Extremlösungsversuchen kommen. Ein gestandener Feuerwehrmann, der bei seinem Einsatz beim ICE-Unglück von Eschede traumatisiert wurde, formulierte es so: «Dann kann man sich gleich die Kugel geben...».
Angst vor Verstärkerverlust und Verlust der Gruppenunterstützung

Die in den bisherigen Abschnitten beschriebenen kognitiven Diskrepanzen gehen möglicherweise mit einem Belastungsgefühl einher. Dies könnte besonders für die durch ein Trauma akut gewordenen Diskrepanzen zwischen Selbst- und Männlichkeitsbild zutreffen. Pennebaker und Kollegen [z.B. Pennebaker et al., 1989] konnten zeigen, daß Belastungsdruck adaptiv dadurch regulierbar ist, die eigene Erschütterung, Betroffenheit und Hilfsbedürftigkeit offen auszusprechen. Bei Frauen, die im Kommunikationsbereich keine geschlechtsrollentypische Barriere haben, führt das Andeuten von Hilfsbedürftigkeit häufig zu Verstärkerzuwachs [Billings und Moos, 1981].

Im Gegensatz dazu ist das Eingestehen der eigenen Erschütterung und Hilfsbedürftigkeit für die A-M typischerweise mit der Antizipation eines Verstärkerverlustes verbunden. A-M nehmen dabei an, in ihrer Bezugsgruppe an Status zu verlieren, kein richtiger Mann mehr zu sein, nicht mehr ernst genommen zu werden usw. Diese Einschätzung ihrer Lage hält die Betreffenden davon ab, sich Kollegen anzuvertrauen oder professionelle Hilfe aufzusuchen. Verbal drückt sich diese Angst einem häufig geäußerten aversiven Begriff wie «Weichei» aus.

Die Möglichkeit, seine eigene Betroffenheit und Hilfsbedürftigkeit beispielsweise in der Gruppe anzusprechen, wird von den A-M typischerweise nicht ausgetestet. Dadurch wird in vielen Fällen die Chance vergeben, die Symptome nicht als endgültiges Versagen der Männlichkeit zu begreifen, sondern als eine vorübergehende Störung der psychischen Gesundheit, zu deren Wiederherstellung man zielgerichtete kollegiale Peer-Group-Hilfe, Notfallhilfe und/oder spezifische psychotherapeutische Hilfe in Anspruch nehmen kann.

\section{Therapeutische Konsequenzen}

\section{Motivierung zur Therapie - Motivierung zu einem veränderten Rollenverständnis}

Abschließend sollen einige Konsequenzen der bisherigen Ausführungen für die kognitive VT skizziert werden, wobei dies aus Platzgründen nur kursorisch geschieht [s. a. Pieper, 1999].

Motivierungsstrategien für die Therapie müssen zunächst am starren Männerbild dieser Patienten ansetzen. Es ist wenig sinnvoll, sie dazu zu drängen, auch die «weiche Seite» in sich zu sehen und zu akzeptieren oder gar sie zu gefühlsmäßigen Statements zu zwingen. Therapieversuche dieser Art, besonders von Therapeutinnen vorgetragen, werden von diesen Männern als Demontage erlebt und verhindern das Zustandekommen eines therapeutischen Vertrauensverhältnisses. 


\section{Psychoedukation}

Statt einer gefühlsbetonten Anfangsphase ist eine psychoedukative Phase wichtig, in der den betroffenen A-M drei Punkte vermittelt werden können:

1. Die Angemessenheit ihrer Reaktionen auf extremen Streß (Normalisierung des Unnormalen: zu erwartende Reaktionen auf eine extrem unnormale Situation)

2. Männer können von extremen Ereignissen genauso traumatisiert werden wie Frauen, verschlechtern jedoch ihre Chance auf Gesundung beträchtlich, wenn sie es zu verbergen versuchen. Das Konzept des A-M wird explizit eingeführt. Es wird anhand von Fallbeispielen vermittelt, welche negativen Verläufe es bei traumatisierten Rettern mit A-M-Merkmalen geben kann.

3. Männer, die es schaffen, ihre Betroffenheit zu verarbeiten, gehen gestärkt aus diesem Prozeß hervor, können Vorbilder für Kollegen werden und dabei helfen, wenn diese ähnliche Probleme nach Einsätzen haben.

Gute Erfahrungen konnten damit gemacht werden, von anderen Feuerwehrleuten, Polizisten zu berichten, die posttraumatische Belastungsstörungen hatten und die in psychologischer Betreuung oder Therapie waren. Dabei wurde meistens Videomaterial eingesetzt, in dem die Betroffenen darüber berichten, daß das Zugeben und Bearbeiten der eigenen Traumatisierung von ihnen selbst und von ihren Kollegen im Nachhinein nicht als Schwäche, sondern als Stärke erlebt wurde.

\section{Gruppeninterventionen}

Noch geeigneter sind spezifisch gestaltete, routinemäßige Gruppeninterventionen (ursprünglicher Begriff «Debriefing») [vgl. aber kritische Diskussion zu Debriefing-Wirksamkeit, Avery und Orner, 1998]. In den von uns eingesetzten Gruppeninterventionen wird der Einsatz (z.B. von Feuerwehrleuten) getrennt auf der kognitiven und auf der emotionalen Ebene mit dem Ziel thematisiert, die persönlichen Erlebnisse und Erfahrungen zu besprechen, deren Verarbeitung $\mathrm{zu}$ forcieren, das Auftreten traumatischer Störungen zu verhindern und wieder einsatzfähig zu werden. Im Ergebnis berichten die Beteiligten, daß es ihnen deutlich leichter fällt, über ihre gefühlsmäßige Beteiligung nachzudenken und sich untereinander auszutauschen. Damit erlernen sie eine aktive Bewältigungsform ihres Traumas, wenn sie an einer solchen Gruppe teilgenommen haben.

Gruppen von traumatisierten Helfern kann durch die Vermittlung des A-M-Risikoprofils verdeutlicht werden, wie gefährlich es ist, Hilfsbedüftigkeit nach Traumatisierungen zu verleugnen. Auf diese Weise können zwei mögliche Effekte erreicht werden:

1. A-M fühlen sich anschließend häufig selbst motiviert, ihre eigene starre Haltung in Frage zu stellen.

2. Kollegen aus dem eigenen Team haben meistens ein feines Gespür dafür, welcher ihrer Kollegen der Gruppe der A-M zuzuordnen ist. Nach der Information über diese Problematik in der psychoedukativen Phase einer Inter- vention sprechen sie häufig von sich aus die betreffenden Personen an und können dadurch eine offenere Haltung für eine aktive Traumabewältigung auslösen.

Das gesamte Klima einer Institution kann sich ändern, wenn eine größere Anzahl der Kollegen an Gruppeninterventionen teilnimmt. Diese Entwicklung konnte z.B. bei den Bediensteten der Justizvollzugsanstalten des Landes Brandenburg festgestellt werden, in denen der eine Autor (G.P.) seit einiger Zeit Gruppeninterventionen eingeführt hat. Das dabei eingesetzte Protokoll «BÜTS» (BÜTS = Bewältigung von Übergriffen und traumatischem Streß) wird regelmäßig durchgeführt, wenn es zu Übergriffen von Gefangenen auf Bedienstete oder $\mathrm{zu}$ anderen traumatischen Ereignissen im Gefängnis kommt (Pieper, 1999).

Die anfänglich ablehnende Haltung vieler Bediensteter in Justizvollzugsanstalten, von denen viele zum Typ der A-M gezählt werden können, über traumatische Ereignisse zu reden wie «Mit so etwas komme ich selber klar...», «Dafür werden wir schließlich bezahlt...» ist inzwischen spürbar einem Klima gewichen, in dem sich auch die Kollegen untereinander offen befragen (kollegiale Peer-Group-Hilfe), wie es dem Einzelnen nach einem dramatischen Vorfall geht.

Auf den Punkt brachte es ein Bediensteter, der anfangs sehr skeptisch war, mit der Feststellung: «Früher haben wir das mit zwei, drei Cognacs weggespült, heute reden wir drüber. Das dauert zwar länger, aber es hilft besser!»

\section{Expositionsbehandlung}

Es soll nur kurz angedeutet werden, daß im Rahmen der regulären psychotherapeutischen Einzelarbeit, bei A-M die Expositionsbehandlung besonders geeignet ist [vgl. Pieper, 1997; für ausführlichere Diskussionen der Expositionstherapie vgl. z.B. Foa et al., 1999]. Bei der Expositionsbehandlung ist die Bereitschaft der A-M, mitzuarbeiten, in der Regel schnell erreicht, da es primär um aktive Handlungen und nicht nur um Gespräche geht. Allerdings ist bei der Therapiedurchführung häufiger zu beobachten, daß sie versuchen, die aufkommende Angst zu unterdrücken bzw. sie nicht vor sich und den Therapeuten zuzugeben. Können sie es erst einmal annehmen, daß eine wahre Leistung darin besteht, eine sich selbst eingestandene Angst zu überwinden, statt sie nur zu negieren und durch Vermeidungsverhalten zu umgehen, empfinden A-M die Expsotionsbehandlung häufig als Wiederherstellung ihrer Männlichkeit mit einem erweiterten Rollenverständnis. Empirische Befunde zur besonderen Passung der A-M-Gruppe für die Expositionstherapie stehen allerdings noch aus.

\section{Ausblick}

Durch klinische Beobachtungen haben wir gezeigt, daß eine Gruppe von Männern in Berufen, in denen Traumatisierungen häufig vorkommen, typischerweise durch das Risikoprofil eines Alpha-Mannes beschreibbar ist. Das beschriebene Risi- 
koprofil sollte durch empirische Untersuchungen weiter überprüft werden, bevor es als gesichertes Wissen gelten kann bisher haben die hier skizzierten Annahmen ausschließlich hypothesen- und diskussionsanregenden Charakter.

A-M haben demnach eine deutlich geringere Motivation und wesentlich höhere innere und äußere Hürden, nach Traumatisierungen und der Ausbildung von posttraumatischen Belastungsstörungen therapeutische Hilfen in Anspruch zu nehmen. Sie wehren sich gegen ein Anerkennen der eigenen Betroffenheit und versuchen sich mit «Scheinlösungen» über Wasser zu halten. Auf diese Weise kommt es zu verspätetem Ausbruch der Symptomatik, schwierigeren Verläufen der PTBS, Chronifizierungen, radikalen Lösungen bis hin zu Suizid oder verfrühtem Tod. Durch die Vermittlung des beschriebenen Therapierationals kann das besonders gefährdete Klientel der A-M «aus der Reserve gelockt» und zur Bearbeitung ihrer traumatischen Erfahrungen angehalten werden.

Diejenigen A-M, die mit therapeutischer Hilfe erfolgreich eine posttraumatische Belastungsstörung bewältigt haben, können vorbildhaft tätig sein in der Aufklärung und Vorbereitung von Kollegen über den Umgang mit traumatischen Situationen. Dazu abschließend noch zwei Beispiele.

Der im Fall 1 beschriebene suizidale Feuerwehrmann etwa hat es sich zur Aufgabe gemacht, nach jedem Feuerwehreinsatz bei jüngeren Kollegen für die Gesprächsangebote und Nachbesprechungen vor Ort bereit zu stehen.

Der im Fall 2 vorgestellte Grubenwehrmann sieht es heute bei Einsätzen als seine wichtigste Aufgabe an, eingeklemmten und verletzten Personen beizustehen. Dies empfindet er als eine Konsequenz aus seinem in Borken erlebten Trauma und aus der Erkenntnis, daß das Leid eines Traumatisierten besonders stark wird, wenn er es sich als «harter Mann» nicht zugestehen kann.

Durch das Trainieren des Redens über die eigene Betroffenheit läßt sich in Berufsgruppen, die oft von den Männeridealen der A-M geprägt sind, eine Kultur entwickeln, die Chancen dafür bietet, daß Männer sich nach dem Erleben eines Traumas rechtzeitig um Hilfe kümmern und damit eine konstruktive Traumabewältigung leisten.

\section{Literatur}

Alfermann D: Geschlechterrollen und geschlechtstypisches Verhalten. Stuttgart, Kohlhammer, 1996. American Psychiatric Association: Diagnostic and Statistical Manual of Mental Disorders (4th ed). Washington, American Psychiatric Association, 1994.

Appel-Schumacher T: Streßmanagement nach traumatischen Ereignissen; in Bengel J (Hrsg): Psychologie in Notfallmedizin und Rettungsdienst. Berlin, Springer, 1997, pp 255-268.

Arbeitsgruppe Stolzenbachhilfe (Hrsg): Nach der Katastrophe. Das Grubenunglück von Borken. Göttingen, Vandenhoek und Ruprecht, 1995.

Avery A, Orner R: First report of psychological debriefing abandoned - The end of an era? International Society of Traumatic Stress Studies News, 1998;2: 3-4. Baider L, Perez T, Kaplan De-Nour A: Der Einfluß des Traumas auf die Bewältigung von Krebs: Überlebende des Holocaust; in Heim E, Perrez M (Hrsg): Jahrbuch Med Psychol 10: Krankheitsverarbeitung. Göttingen, Hogrefe, 1994, pp 117-126.

Bengel J (Hrsg): Psychologie in Notfallmedizin und Rettungsdienst. Berlin, Springer, 1997.

Billings AG, Moos RH: The role of coping responses and social resources in attenuating the stress of life events. J Behavior Med 1981;4:139-157.

Bussey K, Bandura A: Gender constancy, social power, and sex-linked modeling. J Personal Soc Psychol 1984;47:1292-1302.

Butollo W: Traumatherapie. Die Bewältigung schwerer posttraumatischer Störungen. München, CIP Medien, 1997.
Cantor N, Mischel W, Schwartz J: A prototyp analysis of psychological situations. Cognitive Psychol 1982; 14:45-77.

Foa EB, Kozak MJ: Clinical applications of bioinformational theory: Understanding anxiety and its treatment. Behavior Therapy 1998;29:675-690.

Foa EB, Rothbaum BO, Maercker A: Posttraumatische Belastungsstörung; in Margraf $\mathrm{J}$ (Hrsg): Lehrbuch der Verhaltenstherapie (2. Aufl.). Berlin, Springer, 1999.

Janoff-Bulman R: The aftermath of victimisation: Rebuilding shattered assumptions; in Figley CR (ed): Trauma and its Wake. New York, Brunner/Mazel, 1985, pp 15-35.

Jatzko H, Jatzko S, Seidlitz H: Das durchstoßene Herz. Ramstein 1988. Edewecht, Stumpf \& Kossendey, 1995. Kirmayer LJ: Confusion of the senses. Implications of ethnocultural variations in somatoform and dissociative disorders for PTSD; in Marsella AJ, Friedman MJ, Gerrity ET, Scurfield RM (eds): Ethnocultural Aspects of PTSD. Washington, Am Psychol Assoc, 1996, pp 131-163.

Maercker A: Extrembelastungen ohne psychische Folgeschäden: Gesundheitspsychologische Konzepte und Befunde; in Schüffel W (Hrsg): Handbuch der Salutogenese. Wiesbaden, Ullstein, 1998, pp 341-350.

Maercker A: Posttraumatische Belastungsstörungen. Psychologie der Extrembelastungsfolgen bei Opfern politischer Gewalt. Lengerich, Pabst, 1998.

McFarlane AC: Epidemiological evidence about the relationship between PTSD and alcohol abuse: the nature of the association. J Addictive Behaviors, 1998; 23:813-825.
Mitchell JT, Everly GS: Critical Incident Stress Debriefing (CISD) and the prevention of work-related traumatic stress among high risk occupational groups; in Everly GS, Lating JM (eds): Psychotraumatology. New York, Plenum, 1995.

Pennebaker JW, Barger SD, Tiebault J: Disclosure of traumas and health among Holocaust survivors. Psychosom Med 1989;51:577-589.

Pieper G: Betreuung von Katastrophenopfern; in Maercker A (Hrsg): Therapie der posttraumatischen Belastungsstörungen. Berlin, Springer, 1997, pp 251274

Pieper G: Bewältigung von Übergriffen und traumatischem Streß (BÜTS) für Justizvollzugsbedienstete. Z Strafvollzug 4/99 (im Druck).

Raphael B, Wilson J, Meldrom L, McFarlane A: Acute preventive interventions; in van der Kolk BA (ed): Traumatic Stress. New York, Guilford, 1996, pp 463 479.

Schnurr P: Trauma, PTSD, and physical health. PTSD Res Quart 1996;7:1-6.

Sieverding M: Die Bedeutung von Prototype-Matching für präventives Verhalten: Ist die Teilnahme an Streßbewältigungs-Kursen «unmännlich»? Z Gesundheitspsychol 1997;5:272-298.

Ursano R, McCaughey B, Fullertoon C (eds): Trauma and Disaster. The Structure of Human Chaos. Cambridge, Cambridge University Press, 1994.

Wolfe J, Kimerling R: Gender issues in the assessment of posttraumatic stress disorder; in Wilson JP, Keane TM (eds): Assessing Psychological Trauma and PTSD. New York, Guilford, 1997. 